\title{
Newspaper coverage of psychiatry and general medicine: comparing tabloids with broadsheets
}

\section{AIMS AND METHOD}

To study whether psychiatry received differing treatment in newspapers than the other medical specialities, and whether tabloids were more negative in their coverage of general medicine and psychiatry than broadsheets. We conducted a survey of all headlines in all daily newspapers in the Dutch-speaking part of Belgium over a one-month period and judged whether the content was essentially positive, neutral or negative.

\author{
RESULTS \\ There was no significant difference \\ between articles on general medicine \\ and psychiatry with respect to the \\ tone of the article. Whereas negative \\ articles about medicine focused on \\ both doctor and patient to the same \\ extent, negative articles on \\ psychiatry tended more often to \\ describe the patient. Broadsheets \\ published more negative than \\ positive articles, while tabloids \\ published an equal proportion of \\ negative and positive articles.
}

\begin{abstract}
CLINICAL IMPLICATIONS
There appears to be no difference in tone with respect to articles on general medicine and psychiatry, respectively. Nevertheless, the fact that negative articles on psychiatry tend to focus more exclusively on the patient points towards continuing stigmatisation of the psychiatric patient. Finally, broadsheets tend to be more negative in covering both general medicine and psychiatry, which contrasts with earlier findings.
\end{abstract}

Negative press coverage has been thought to play a role in the stigmatising attitudes towards mental illness in the Western world. This seems to be more so in tabloids than in broadsheets (Peters, 2002). Doctors in general also seem to get a 'bad press'. The frequency of articles about health seems to increase over time, but the ratio of negative to positive articles has remained unchanged in UK broadsheets over the past 21 years (Ali et al, 2001). While good news and bad news were equally likely to be featured in the press releases of scientific journals, bad news was more likely to be reported in newspaper articles (Bartlett et al, 2002). In this study, replicating work by Lawrie (2000), we wanted to investigate whether psychiatry received worse treatment than other medical specialities in Flemish newspapers. Furthermore, we wanted to test the hypothesis that tabloids are more negative in their coverage of general medicine and psychiatry.

\section{Method}

All daily newspapers in Flanders, the Dutch-speaking, northern part of Belgium (population: 6000 000) were monitored for one month (November 2000). All article headlines about health-related issues were included in this survey. Of eight newspapers surveyed, three target a more educated public and are referred to as 'broadsheets' (combined circulation of 225000 ) and five are 'tabloids', aimed at the general public (combined circulation of 912000 ).

Four researchers made group consensus judgements as to whether the headlines were about psychiatric or general medical issues and whether they were mainly positive, neutral or negative in tone. It should be borne in mind that this judgement was made from the headline, which not always adequately reflects the tone of the piece (O'Connor \& Casey, 2001): sometimes the piece was neutral, but the headline had been devised insensitively or even crudely. Examples of headlines about general medicine are: 'Comatose patients are being dumped' (negative), 'Hospital opens ward for stroke patients' (positive) and 'Why do we sneeze?' (neutral). 'I'd rather wander in the streets' was considered a negative message about psychiatry, while 'Horses help to conquer psychiatric disorder' and 'Relation between fingers and sexual inclination' were scored as positive and neutral, respectively for psychiatry.

Furthermore, a distinction was made with respect to subject matter: patients, doctors/therapists, and a 'general' rest category, including organisation of care.

\section{Results}

Five hundred health-related article headlines were collected. Of these, 380 were about general medicine (76\%), of which an equal proportion were judged to be negative (35\%), positive (30\%) and neutral $(35 \%)$ in tone. One hundred and twenty headlines concerned psychiatry, of which $38(32 \%)$ were considered to be negative, 36 (30\%) positive and $46(38 \%)$ neutral in tone.

The article headlines judged to be neutral in tone were excluded from the following analyses. The total number of headlines judged to be either positive or negative was 320 . There was no significant difference between articles on general medicine and articles on psychiatry with respect to the tone of the article (ratio of positive vs. negative articles on general medicine: 112/ 134; ratio of positive vs. negative articles on psychiatry: 36/38; $\left.\chi^{2}=0.223, d f=1, P=0.64\right)$. There were, however, differences with respect to subject matter. Negative articles about medicine focused on both the doctor and the patient to the same extent (ratio of positive vs. negative articles focusing on the doctor: 56/64; ratio of positive vs. negative articles focusing on the patient: 
7

original

papers
15/15; $\left.\chi^{2}=0.107, d f=1, P=0.74\right)$. Negative articles on psychiatry, however, tended to describe the patient (ratio of positive vs. negative articles focusing on the doctor: 19/5; ratio of positive vs. negative articles focusing on the patient: $7 / 17 ; \chi^{2}=12.084, \mathrm{df}=1, P=0.001$ ).

Overall, our data showed a significantly different proportion of positive to negative articles in the tabloids than in the broadsheets. The latter published significantly more articles that were negative in tone, whereas the former published an equal number of negative and positive articles (ratio of positive vs. negative articles in the tabloids: 102/94; ratio of positive vs. negative articles in the broadsheets: $46 / 78 ; \chi^{2}=6.823, \mathrm{df}=1$, $P=0.009$ ). The same tendency was visible in the articles on psychiatry, but due to the small sample size, this difference did not reach statistical significance.

\section{Discussion}

A quarter of all article headlines about medical matters published in Flemish newspapers in November 2000 dealt with psychiatric subjects. This proportion is somewhat higher than that found by Lawrie (2000) in nine UK dailies. The positive to negative tone ratio in the Flemish study also seems higher than that in similar studies in the UK (Ali et al, 2001; Lawrie, 2000) and Canada (Day \& Page, 1986), and this holds for both general medicine and psychiatry. Furthermore, this study failed to confirm findings of the UK study that revealed a more negative coverage of psychiatry in comparison with the rest of medicine (Lawrie, 2000). Our finding that negative articles on psychiatry were more focused on patients is in agreement with the UK study. This in turn corroborates the idea that newspapers play a role in the continuing stigmatisation of psychiatric patients.

The fact that tabloids tend to write more favourably on medicine than broadsheets came as a surprise, and runs contrary to UK findings. The fact that Flemish tabloids are clearly less 'sensational' than (some of) the UK tabloids might partly account for this finding. In addition, the Flemish press is, in general, more reserved when covering sensitive topics.

In conclusion, there seems to be as much positive as negative reporting on medical and psychiatric issues in Flemish dailies. This is not only in contrast with earlier findings, but also with the fact that psychiatrists often have the impression that themselves, psychiatric patients and psychiatry in general are negatively pictured in newspapers. One explanation for this discrepancy could be that the public has indeed become more critical of the medical establishment, which may be reflected in the press. This in turn could explain why broadsheets tend to focus more on negative messages and pay less attention to positive ones. Another plausible explanation could be that doctors are more sensitive to negative than to positive messages in the press with respect to their profession, and therefore simply get the wrong impression. It is generally not the psychiatrist but the psychiatric patient who gets a bad press. Therefore, media campaigns against the stigmatisation of psychiatric patients should be encouraged.

Press coverage of general medical and psychiatric matters may be highly dependent on language and culture. The fact that our findings run contrary to earlier (British and Canadian) findings in a number of ways, implies that with respect to press coverage of medical matters, a cross-cultural perspective should be adopted, and cross-cultural studies should be encouraged.

\section{Acknowledgements}

Tijl Callewaert, MD and Steven Vandenbosch, MD, both psychiatric trainees in the University Center St Jozef in Kortenberg at the time, contributed to this article by gathering data and participating in the discussion about the results.

\section{References}

ALI, N.Y., LO,T.Y. S., AUVACHE, V., et al (2001) Bad press for doctors: a 21 year survey of three national newspapers. BMJ, 323, 782-783.

LAWRIE, S. M. (2000) Newspaper coverage of psychiatric and physical illness. Psychiatric Bulletin, 24, 104-106.

BARTLETT, J., STERNE, J. \& EGGER, M. (2002) What is newsworthy? Longitudinal study of the reporting of medical research in two British newspapers. BMJ, 325, 81-84.

DAY, D. M. \& PAGE, S. (1986) Portrayal of mental illness in Canadian O'CONNOR, A. \& CASEY, P. (2001) What it says in the papers: an audit. Irish Journal of Psychological Medicine, 18, $68-71$.

PETERS, C. (2002) Doctors' bad press depends on type of newspaper. BMJ, 324, 241 (Letter).

Psychiatry, 31, 813-817.

${ }^{*}$ Guido Pieters Head of BehaviourTherapy Department, University Center St Jozef Kortenberg, Leuvansesteenweg 517, B3070 Kortenberg, Belgium Guido.Pieters@med.kuleven.ac.be, Véronique De Gucht Researcher, Department of Clinical Psychology, University Center St Jozef Kortenberg and Assistant Professor, Department of Clinical and Health Psychology, Leiden University, The Netherlands, Hendrick Kajosch Psychiatric Trainee, University Center St Jozef Kortenberg 